Int. J. Dev. Biol. 61: 347-356 (2017)

doi: $10.1387 / \mathrm{ijdb} .160331 \mathrm{sb}$

\title{
Regulatory heterochronies and loose temporal scaling between sea star and sea urchin regulatory circuits
}

\author{
TSVIA GILDOR ${ }^{1}$, VERONICA HINMAN² and SMADAR BEN-TABOU-DE-LEON*,1 \\ ${ }^{1}$ Department of Marine Biology, Leon H. Charney School of Marine Sciences, University of Haifa, Haifa, Israel and \\ ${ }^{2}$ Departments of Biological Sciences and Computational Biology, Carnegie Mellon University, Pittsburgh, PA, USA
}

\begin{abstract}
It has long been argued that heterochrony, a change in relative timing of a developmental process, is a major source of evolutionary innovation. Heterochronic changes of regulatory gene activation could be the underlying molecular mechanism driving heterochronic changes through evolution. Here, we compare the temporal expression profiles of key regulatory circuits between sea urchin and sea star, representative of two classes of Echinoderms that shared a common ancestor about $\mathbf{5 0 0}$ million years ago. The morphologies of the sea urchin and sea star embryos are largely comparable, yet, differences in certain mesodermal cell types and ectodermal patterning result in distinct larval body plans. We generated high resolution temporal profiles of 17 mesodermallyendodermally- and ectodermally-expressed regulatory genes in the sea star, Patiria miniata, and compared these to their orthologs in the Mediterranean sea urchin, Paracentrotus lividus. We found that the maternal to zygotic transition is delayed in the sea star compared to the sea urchin, in agreement with the longer cleavage stage in the sea star. Interestingly, the order of gene activation shows the highest variation in the relatively diverged mesodermal circuit, while the correlations of expression dynamics are the highest in the strongly conserved endodermal circuit. We detected loose scaling of the developmental rates of these species and observed interspecies heterochronies within all studied regulatory circuits. Thus, after $\mathbf{5 0 0}$ million years of parallel evolution, mild heterochronies between the species are frequently observed and the tight temporal scaling observed for closely related species no longer holds.
\end{abstract}

KEY WORDS: evolution, heterochrony, temporal scaling, gene regulatory network, echinoderm, expression dynamics

\section{Introduction}

In 1875, the zoologist, Ernst Haeckel, introduced the concept of heterochrony, a relative change in timing of a developmental process during evolution (Haeckel, 1875). Years later, the evolutionary embryologist, de Beer, proposed that heterochrony is a major source of evolutionary innovation contributing to the origin of many taxa (De Beer, 1930). This idea was further developed by Gould and discussed by both evolutionary and developmental biologists (Gould, 1977; Raff, 1996). During the molecular age, the concept of heterochrony transformed to describe evolutionary change in the timing of gene activation that consequently alters the developmental program (Abrahante et al., 1998; Pasquinelli et al., 2000; Sakamoto et al., 2009). There are now several examples of heterochronic changes in gene activation that possibly underlie the evolutionary differences between related species (Gunter et al., 2014; Morino et al., 2012; Sakamoto et al., 2009; Smith et al., 2008). These studies are illuminating, but they focus on a small set of genes and are not framed within the context of a gene regulatory network. Comparative studies of gene initiation times within regulatory circuits could establish the prevalence of heterochronic changes and how such changes influence embryo morphology.

Recent studies have shown that heterochrony is not common in closely related species that have similar body plans (Gildor and Ben-Tabou De-Leon, 2015; Levin et al., 2012; Yanai et al., 2011). The developmental transcriptomes of related species that

Abbreviations used in this paper: EMT, epithelial to mesenchymal transition; GRN, gene regulatory network.

\footnotetext{
*Address correspondence to: Smadar Ben-Tabou de-Leon. Department of Marine Biology, Leon H. Charney School of Marine Sciences, University of Haifa, Haifa 31905, Israel. Fax:+972-4-8288267.Tel: +972-4-8288555. E-mail: sben-tab @ univ.haifa.ac.il - web: http://marsci.haifa.ac.il/research/research-laboratories-and-projects/ marine-biology-laboratories/gene-regulation-in-development-and-evolution - (iD) http://orcid.org/0000-0001-9497-4938
} 


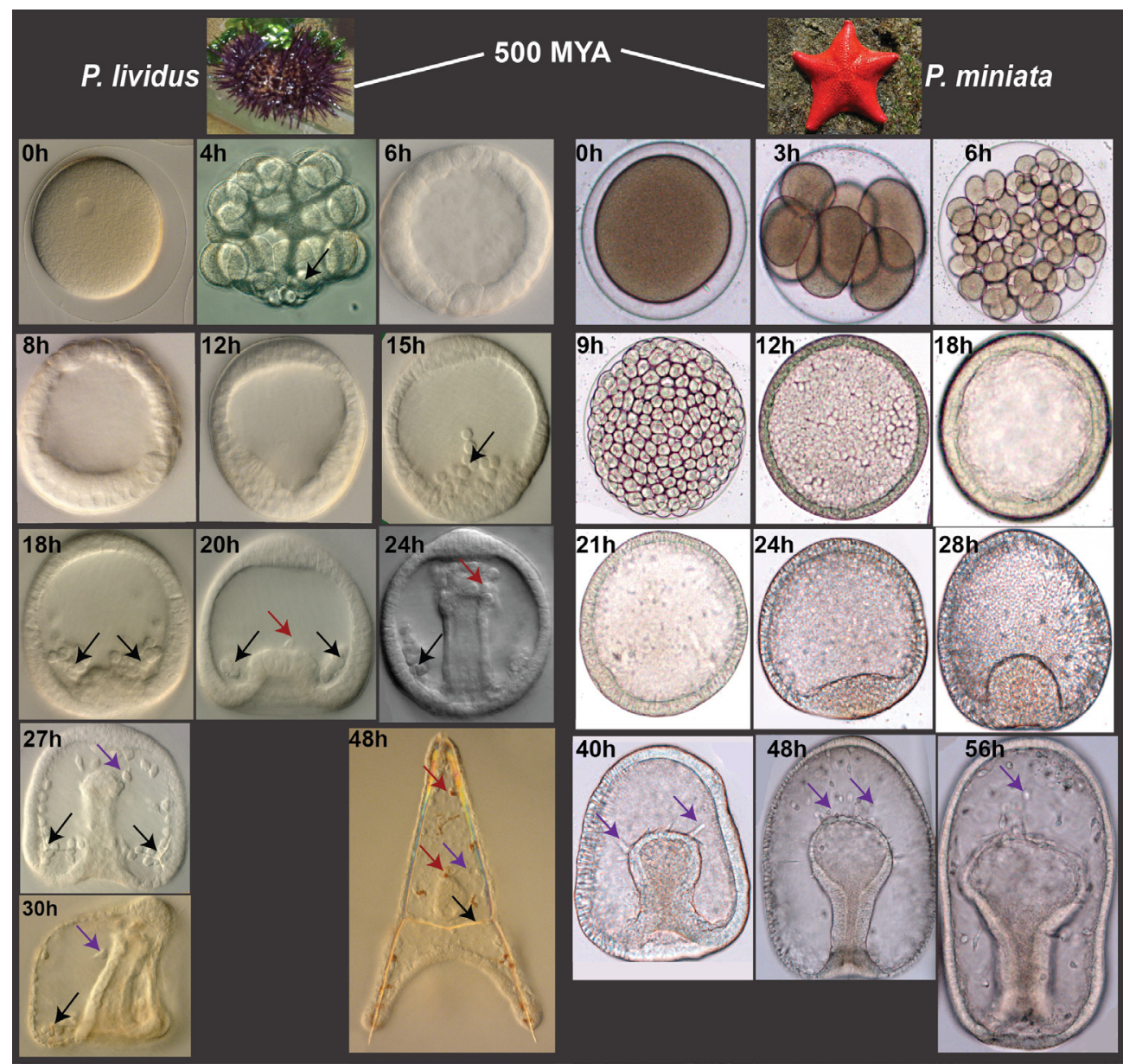

Fig. 1. $\boldsymbol{P}$ lividus and $\boldsymbol{P}$ miniata development. Black arrows indicate the sea urchin skeletogenic cells brown arrows indicate the sea urchin pigment cells and purple arrows indicate the sea urchin and sea star blastocoelar cells. Time is indicated as hours post fertilization in each figure. See text for further details. similarities in gut invagination (Fig. 1). The mesodermal blastocoelar lineage goes through epithelial to mesenchymal transition (EMT) and ingress into the blastocoel at late gastrula in both species (purple arrows in Fig. 1). The skeletogenic lineage and the pigment cells are two mesodermal lineages, specific to the sea urchin, that go through EMT earlier in development. The skeletogenic mesodermal cells ingress into the blastocoel at swimming blastula stage and generate the larval skeleton that gives the sea urchin its pluteus morphology (black arrows in Fig. 1). The pigment mesodermal cells start their ingression at early gastrula and give the sea urchin larva its red pigmentation (brown arrows in Figs. 1). The early ingression of these two novel lineages suggests that a change in the timing of gene activation may have been a part of the regulatory changes that lead to their evolution in the sea urchin embryo. Other differences were identified in ectodermal patterning, likely associated with the formation of two ciliary bands that transverse the ectoderm in sea stars, compared to the single ciliary band in sea urchins (Yankura et al., 2010). Thus, the overall larval body plan is comparable between the sea star and sea urchin but the evolution of novel mesodermal cell lineages and changes in ectodermal are morphologically similar revealed a global temporal scaling of gene initiation timing with very few cases of heterochrony (Levin et al., 2012; Yanai et al., 2011). Scaling the developmental rates of two sea urchin species that have similar morphology showed striking conservation of gene initiation timing and overall expression dynamics of regulatory genes (Gildor and Ben-Tabou De-Leon, 2015). Changes in gene expression levels, defined as heterometries, were more frequent than heterochronic changes yet seemed to be buffered by the developmental program (Gildor and Ben-Tabou De-Leon, 2015; Yanai et al., 2011). Thus, conservation of morphology seems to require precise temporal scaling of gene initiation times between related species. Will this tight scaling and the rareness of heterochrony be observed in larger evolutionary distances, where there is a noticeable change in the body plan?

The sea urchin, e.g., Strongylocentrotus purpuratus (S. purpuratus) or Paracentrotus lividus ( $P$. lividus), and the sea star, Patiria miniata ( $P$. miniata) shared a common ancestor about 500 million years ago (Smith et al., 2013) and their larval body plans show some divergence (Hinman and Davidson, 2007; Hinman et al., 2003). The sea star, P. miniata egg is larger than that of the sea urchin $(\sim 200 \mu \mathrm{m}$ compared to $\sim 70 \mu \mathrm{m}$, Fig. 1) and the duration of the cleavage stage is longer than in the sea urchin (Fig. 1). Later in development, the embryonic body plans have general patterning led to observable morphological differences between the larvae of the two species.

The models of the gene regulatory networks (GRNs) that control sea urchin embryonic development are highly comprehensive (BenTabou De-Leon et al., 2013; Li et al., 2012; Oliveri et al., 2008) and so are the models of the sea star developmental GRNs (Hinman et al., 2003; Mccauley et al., 2013; Mccauley et al., 2010; Mccauley et al., 2012; Yankura et al., 2013; Yankura et al., 2010). Some of the regulatory circuits of these models show high conservation of the spatial expression and linkages, but there are also noted differences (Fig. 2, (Hinman and Davidson, 2007; Hinman et al., 2003; Mccauley et al., 2010; Mccauley et al., 2012). In the sea star, the ectoderm is extensively patterned along the major embryonic axis, the animal-vegetal axis, while in the sea urchin the ectodermal patterning are compacted at the animal pole (Yankura et al., 2010). Furthermore, certain differences between the species exist in specification of the animal-most sensory neurons (Yankura et al., 2013). The endomesoderm specification in both species is initiated by high levels of nuclear $\beta$-catenin (Logan et al., 1999; Mccauley et al., 2015; Peter and Davidson, 2011b). In the sea star, high nuclear $\beta$-catenin concentrations are required for mesodermal specification and lower levels for endoderm specification (Mccauley et al., 2015) while in the sea urchin nuclear $\beta$-catenin 
is directly activating endodermal genes (Ben-Tabou de-Leon and Davidson, 2010; Peter and Davidson, 2011b). Additionally, in the sea urchin there is spatial exclusion of mesodermal regulatory gene expression into three distinct cell lineages, the blastocoelar cells, the skeletogenic lineage and the pigment cells, of which only the blastocoel cells are observed in the sea star (Mccauley et al., 2012). This exclusion has possibly resulted from a change in the mesodermal GRN connectivity that induced mutual repression between different regulatory genes and supported the evolution of the two novel mesodermal lineages. Yet, despite the early differences in GRN initiation there is also significant conservation of GRN structure and components between the sea star and the sea urchin in all embryonic territories (Fig. 2).

Here we sought to study the temporal profiles of key regulatory circuits in the sea urchin and the sea star to determine whether there is temporal scaling or regulatory heterochronies between these species. We generated high resolution temporal expression profiles of 17 regulatory genes that control mesoderm, endoderm and ectoderm cell fate specification in the sea star, P. miniata. We then compared gene initiation times and the temporal kinetics to those of their orthologs in the Mediterranean sea urchin, $P$. lividus using the approach we previously developed for comparing two sea urchin species (Gildor and Ben-Tabou De-Leon, 2015). We identify heterochronic changes of gene activation in all embryonic territories, but the order of gene activation is more variable within the mesodermal territory. The time window in which most genes are turned on is equivalent in the different territories within each species and allows for loose scaling of the species developmental rates. Changes in relative levels between the zygotic and maternal phases of gene activity are also observed. Both observed heterochronies, and relative level changes, may have contributed to the differences in the larval body plan between the two organisms.

\section{Results}

\section{Structure of selected mesodermal, endodermal andectodermal gene regulatory circuits in $\mathrm{P}$. miniata and $\mathrm{P}$. lividus}

To study regulatory heterochrony and temporal scaling, we wanted to compare the temporal profiles of regulatory genes that are expressed in largely equivalent spatial domains of the sea urchin and sea star embryos. This is since a change of the spatial domain of gene expression could go beyond a simple change in
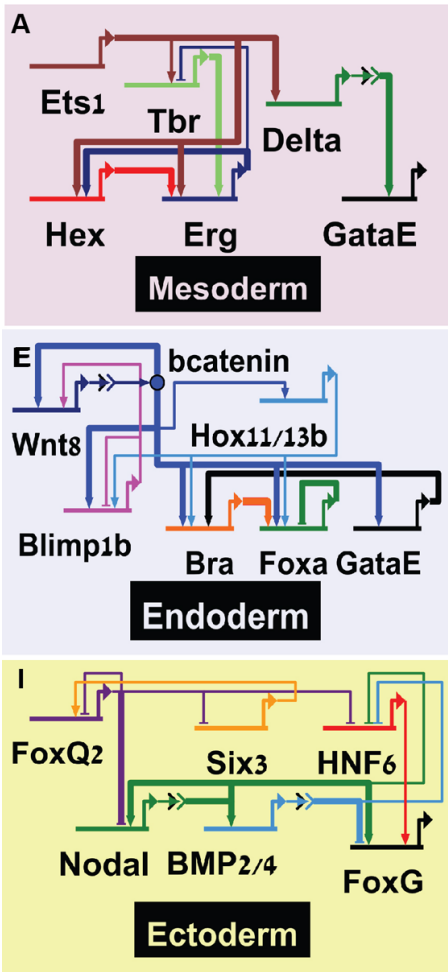
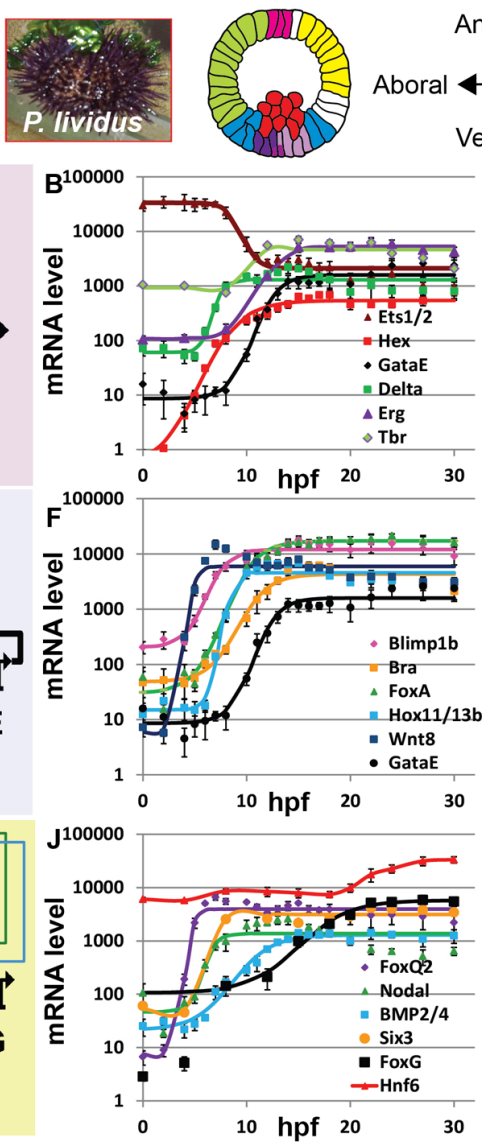
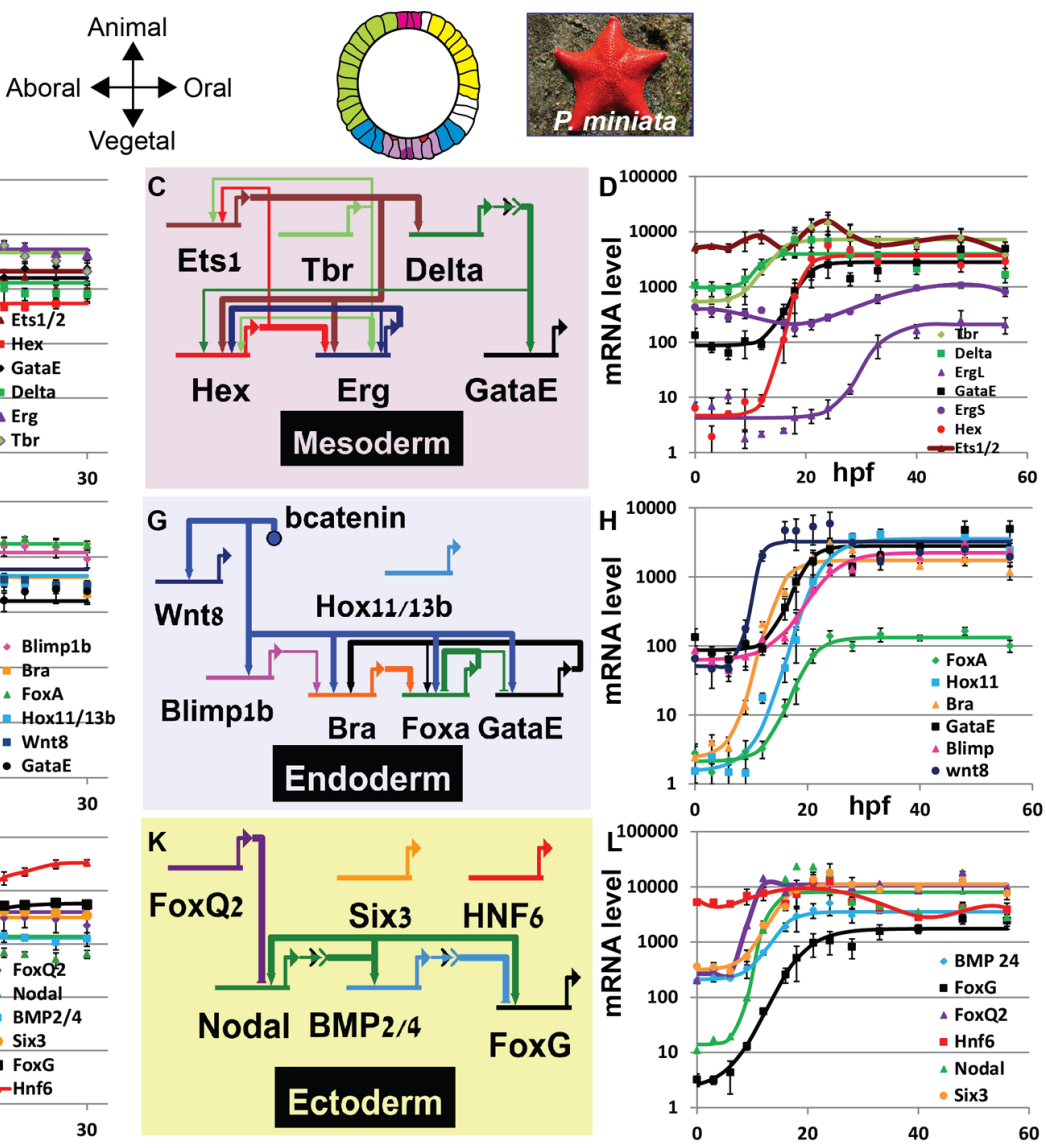

Fig. 2. Regulatory circuit diagrams and temporal profiles of key regulatory genes in the sea urchin and the sea star. Each node in the diagrams represents the cis-regulatory modules of a gene. An arrow going out of a node indicates the protein that the gene encodes and its regulatory activity: transcriptional activation is indicated by arrow, transcriptional repression is indicated by horizontal bar. Color code is similar to genes in both species and to the gene temporal profiles. Conserved links are thick and links that are species specific are thin. Expression profiles were measured by QPCR and fitted when possible by sigmoidal fit; each time point is the average of three independent biological replicates. Error bars indicate standard deviation. $A, E$, and I, P. lividus mesoderm, endoderm and ectoderm regulatory circuits, respectively. B, F and J, temporal expression profiles of P. lividus mesodermal, endodermal and ectodermal genes, respectively. C, G and K, P. miniata mesoderm, endoderm and ectoderm regulatory circuits, respectively. $D, H$ and $L$, temporal expression profiles of P. miniata mesodermal, endodermal and ectodermal genes, respectively. 
timing of gene expression that we wish to focus on here. Hence, we did not include the gene encoding the transcription factor GCM, that is critical for the mesodermal pigment lineage specification in the sea urchin, since in the sea star this gene is expressed in the ectoderm (Ransick and Davidson, 2006; Yankura et al., 2013). We also did not include the sea urchin skeletogenic specific genes, e.g., the repressor Pmar1 and the signaling genes VEGF and VEGFR that are not expressed in the sea star embryo (AdomakoAnkomah and Ettensohn, 2013; Duloquin et al., 2007; Morino et al., 2012). In both organisms the germ layers are specified along the animal-vegetal axis, the endomesoderm at the vegetal half and the ectoderm at the animal half (Fig. 2). We selected genes from the three embryonic germ layers and extracted circuit connectivity from published data.

We selected orthologs of five transcription factors and one signaling ligand expressed in the mesodermal cells of the sea urchin (Fig. 2A, (Lee and Davidson, 2004; Materna and Davidson, 2012; Materna et al., 2013; Oliveri et al., 2008)) and the sea star (Fig. 2C (Hinman and Davidson, 2007; Mccauley et al., 2010; Mccauley et al., 2012)). In the sea star, the spatial expression of these genes overlaps at the early stages, while in the sea urchin there is a partial exclusion within the mesodermal lineages. That is, in the sea urchin, the gene that encodes the transcription factor $t b r$ is exclusively expressed in the skeletogenic lineages (red cells in sea urchin diagram, Fig. 2), while Ets1, Hex, Erg are first expressed in the skeletogenic mesoderm and then expand to the blastocoelar cells (light purple cells in both sea urchin and sea star diagrams, Fig. 2 (Materna et al., 2013; Oliveri et al., 2008). The gene that encodes the ligand Delta is first expressed in the skeletogenic cells in the sea urchin, and then turns off in these cells and activated in the pigment cells (marked in dark purple in sea urchin diagram, Fig. 2) and in the blastocoelar cells (Croce and Mcclay, 2010; Materna and Davidson, 2012; Rottinger et al., 2006)). The gene that encodes the transcription factor GataE is expressed in the non-skeletogenic mesodermal cells and then expands to the endoderm in the sea urchin (Materna et al., 2013), similarly, its homolog in the sea star is expressed first in the mesoderm and then in the endoderm (Mccauley et al., 2015). Seven of the twelve regulatory links within the sea star mesodermal circuit are conserved in the sea urchin circuit that has two additional regulatory links (conserved links are thick while species unique links are thin, $2 \mathrm{~A}$ to $2 \mathrm{C}$ ). Thus, despite the novel spatial exclusion in the sea urchin mesoderm, the regulatory linkages within the mesodermal circuits show a noticeable level of conservation after 500 million years of divergence.

In the endodermal circuit we selected five transcription factors and one signaling ligand that are central to the endoderm specification program in the sea urchin (Fig. 2E, (Peter and Davidson, 2011b)) and the sea star (Fig. 2G, (Hinman and Davidson, 2007; Hinman et al., 2003)). In both species these genes are first expressed in the endomesoderm progenitor field and only later in development they clear from the mesodermal cells and stay only in the endodermal cells (Cui et al., 2014; Smith and Davidson, 2008; Smith et al., 2007). The spatial expression and regulatory linkages of the gene hox11/13b was not studied in the sea star. Yet, in the sea urchin this gene plays a central role in the regulation of the endoderm specification and therefore was included in the endodermal diagram (Peter and Davidson, 2011b). In the two organisms, $\beta$-catenin enters the nuclei in the vegetal half of the embryo, binding the transcription factor Tcf to activate expression of endodermal-target genes (Cui et al., 2014; De-Leon and Davidson, 2007; Mccauley et al., 2015). Eight of the 11 regulatory linkages within the sea star endodermal circuit are conserved in the sea urchin. The sea urchin has an additional six regulatory links, four of which are related to the hox11/13b gene that was not studied in the sea star (Fig. 2 E,G). The endodermal regulatory circuit demonstrates the deep conservation of circuit structure between these two organisms.

The ectoderm of the sea urchin is divided into four major territories: the oral ectoderm, where the mouth forms (yellow cells in sea urchin and sea star diagrams, Fig. 2), the aboral ectoderm, (green cells, Fig. 2), the apical domain that at later developmental stages becomes a neurogenic center (magenta cells, Fig. 2), and the ciliary band that separates between the oral and the aboral ectoderm (white cells, Fig. 2). In the sea star, the ciliary band transverses the ectoderm and four spatial ectodermal territories form along the animal-vegetal axis (Yankura et al., 2010). We selected six regulatory genes, key to the ectoderm specification in the sea urchin (Fig. 2I, (Ben-Tabou De-Leon et al., 2013; LI et al., 2014; Saudemont et al., 2010; Wei et al., 2009; Yaguchi et al., 2008)) and the sea star (Fig. 2K, (Yankura et al., 2013; Yankura
Fig. 3. Initiation times in the sea star, $P$ miniata and the sea urchin, $S$. purpuratus, compared to those in the sea urchin, P. lividus. Initiation times and half-life times in each species were estimated using a sigmoid fit on the measured temporal profiles, see methods for details. Endodermal genes are marked in blue, mesodermal genes in red and ectodermal genes in green. Error bars indicate estimated fit error. Black line show the linear regression of the Pm-PI and Sp-PI slopes. The gene foxg was excluded from the linear regression in both graphs since its early spatial expression in P. miniata is different than in the sea urchin. A, Pm-PI initiation times, B, Sp-Pl initiation times.

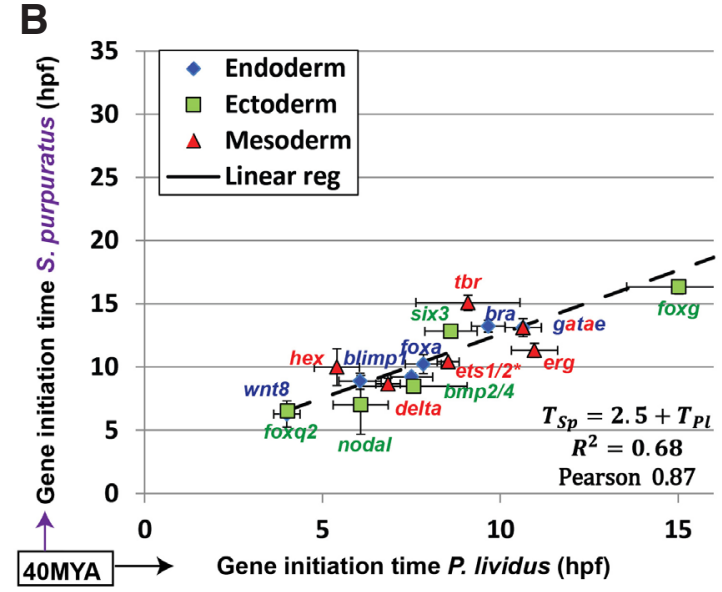

A

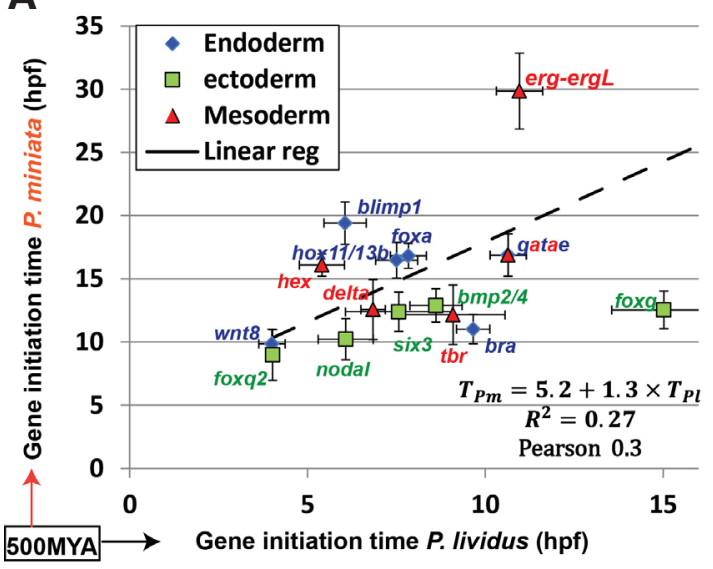


et al., 2010)). The TGF- $\beta$ signaling ligands, Nodal and BMP2/4, are expressed in the oral ectoderm of the two organisms and are the major players in oral-aboral axis formation. Nodal activates the expression of oral ectoderm genes, while BMP2/4 is inhibited in the oral ectoderm and activates genes in the aboral ectoderm ((Ben-Tabou De-Leon et al., 2013; Saudemont et al., 2010). FoxQ2 and Six 3 are transcription factors that control the specification of the apical domain (Wei et al., 2009; Yaguchi et al., 2008; Yankura etal., 2010). The transcription factor HNF6 is maternally expressed and its zygotic expression is exclusive to the ciliary band in both organisms (Barsi and Davidson, 2016; Otim et al., 2005). The transcription factor Foxg is also expressed in the ciliary band in the sea urchin and sea star (Barsi et al., 2015; Mccauley et al., 2015; Tu et al., 2006; Yankura et al., 2013), but in the sea star it has an early expression phase in the mesoderm (Mccauley et al., 2015). The regulatory linkages within the ectodermal territory were extensively studied in the sea urchin leading to 13 regulatory linkages within its circuit diagram, while only 5 regulatory links were verified in the sea star diagram (Fig. $2 \mathrm{I}, \mathrm{K}$ ). Nevertheless, all five regulatory links in the sea star circuit are conserved in the sea urchin diagram, implying at least partial conservation within the ectodermal circuit.

\section{Temporal expression profiles show delay in the maternal to zygotic transition in P. miniata compared to P. lividus}

We studied the temporal expression profiles of the regulatory circuits described above in a developmental window that includes gut invagination and the ingression of all three mesodermal lineages. The overall developmental rate of the sea star, P. miniata is slower than that of the Mediterranean sea urchin, $P$. lividus (Fig. 1). Thus, we studied temporal expression profiles in the sea star from the fertilized egg to 56 hours post fertilization (hpf) and in the Mediterranean sea urchin from Ohpf to $30 \mathrm{hpf}$. For the sea urchin time course we used published data (Gildor and Ben-Tabou de-Leon, 2015) as well as generated new temporal profiles of the genes tbr, egr, six 3, hnf6 and foxg that were not studied there. To generate temporal expression profiles of $P$. miniata and $P$. lividus, we pooled thousands of embryos of each species in different time intervals and measured gene expression levels by quantitative PCR (QPCR, see methods for details). We extracted zygotic gene initiation times and half-life times of maternal genes using a sigmoid fit as described in Gildor and Ben-Tabou de-Leon, 2015 and Gildor et al., 2016. Temporal expression profiles are presented in Fig. 2; measured initiation times in P. miniata and $P$. lividus are presented in Fig. 3A.

The first zygotic genes that turn on in both sea star and sea urchin are wnt8 and foxq2 that are expressed in the two opposing sides of the animal vegetal axis: $w n t 8$ is expressed at the vegetal pole (Cui et al., 2014; McCauley et al., 2015; Fig. 2 F,H), and foxq2 is expressed at the animal pole (Yaguchi et al., 2008; Yankura et al., 2010; Fig. $2 \mathrm{~J}, \mathrm{~L})$. These two genes are expressed at $\sim 4 \mathrm{hpf}$ in $P$. lividus and $\sim 9-10 \mathrm{hpf}$ in P. miniata, and likely mark the onset of the maternal to zygotic transition (Tadros and Lipshitz, 2009; Walser and Lipshitz, 2011). This $\sim 5$ hour delay in the maternal to zygotic transition might be related to the longer cleavage stage observed in the sea star up to $9 \mathrm{hpf}$ compared to the $4 \mathrm{hpf}$ in the sea urchin (Fig. 1). The synchronized activation of these two spatially opposing genes across such immense evolutionary distances suggests that their timely activation must be important to the patterning of the animal-vegetal axis, which both genes contribute to in the two species (Yaguchi et al., 2008; Yankura et al., 2013; Yankura et al., 2010).

\section{Changes in the order of gene activation and relative levels between P. miniata and P. lividus in all regulatory circuits}

After the maternal to zygotic transition, zygotic genes are turned on in all embryonic territories in the two species, and changes in the order of gene activation and relative expression level are observed (Figs. 2, 3). To compare the order of gene activation between P. lividus and P. miniata, we adopted the Velhagen method to analyze developmental sequence (Velhagen, 1997), treating the activation of each gene as a sequence unit. This analysis is summarized in Table 1. The table also includes the order of gene activation in the sea urchin species, S. purpuratus (Materna et al., 2010 ) to demonstrate which sequences are sea urchin consensus and which diverge within sea urchin species.

In the mesodermal circuit, the sea star delta and tbrgenes turn on at about the same time, while in $P$. lividus delta is activated earlier than $t b r$ (compare Fig. 2B and D, Table 1). The gene erg has two isoforms in the sea star; ergS is maternal and ergL is zygotic (Mccauley et al., 2010). ergL and the zygotic phase of ergS are activated much later than all other sea star mesodermal genes, while the P. lividus erg is the last mesodermal gene to be activated, but its time of activation is closer to the activation of the rest of the genes (Fig. 2B and D, Fig. 3A). Possibly, the maternal ErgS protein compensates for the late activation of the zygotic erg genes in the sea star. It is important to note that the order of gene activation shows variation even between the two closely related species, $P$. lividus and $S$. purpuratus, which is not the case in other embryonic territories (Table 1). These results suggest that the mesodermal lineage is less resistant to changes in the order of gene activation compared to the endoderm and the ectoderm lineages.

Another observable regulatory change within the mesodermal circuit is the ratio between the maternal to the zygotic expression level of the gene that encodes the transcription factor Ets $1 / 2$. In both $P$. lividus and $S$. purpuratus the maternal expression level is much higher than the zygotic expression level (Fig. 2B; (Materna et al., 2010), while in the sea star the levels are comparable (Fig. 2D). Ets $1 / 2$ expression is critical to the first steps of skeletogenic

TABLE 1

\section{ORDER OF GENE ACTIVATION IN P. LIVIDUS, S. PURPURATUS AND P. MINIATA}

\begin{tabular}{|c|c|}
\hline \multicolumn{2}{|l|}{ Mesoderm } \\
\hline P. lividus & hex, delta,tbr, gatae-erg \\
\hline S. purpuratus & delta-hex, erg, gatae, tbr \\
\hline P. miniata & tbr-delta, hex-gatae, erg \\
\hline \multicolumn{2}{|l|}{ Endoderm } \\
\hline P. lividus & wnt8, blimp1b, hox11/13b, fox, bra, gatae \\
\hline S. purpuratus & wnt8, blimp1b, hox11/13b, fox, bra-gatae \\
\hline P. miniata & wnt8, bra, hox11/13b, foxa, gatae, blimp1 \\
\hline \multicolumn{2}{|l|}{ Ectoderm } \\
\hline P. lividus & foxq2, nodal,six3, bmp2/4, foxg \\
\hline S. purpuratus & foxq2, nodal. Six3, bmp2/4, foxg \\
\hline P. miniata & foxq2, nodal,six3-bmp2/4-foxg \\
\hline
\end{tabular}

A difference of less than half an hour in the time of activation is below our temporal resolution and we indicate it by a dash connecting the genes (e.g, gatae-erg in P. lividus). In bold we mark genes that show interspecies differences in their activation order. 
differentiation in the sea urchin embryo and is an activating input into most skeletogenic regulatory and differentiation genes (Oliveri et al., 2008). Possibly, the increase in the maternal expression level of this gene in the sea urchin contributes to the early activation of the skeletogenic regulatory circuit.

Changes in the order of gene activation between the sea urchin and sea star are also observed in the endoderm circuit that shows consensus within sea urchin species (Table 1). In the sea star endoderm, bra is the next gene to turn on after wnt8, while in the sea urchin, bra turns on only after blimp1, hox11/13b and foxa turn on (Fig. 2 F,H, Table 1). This difference may be due to
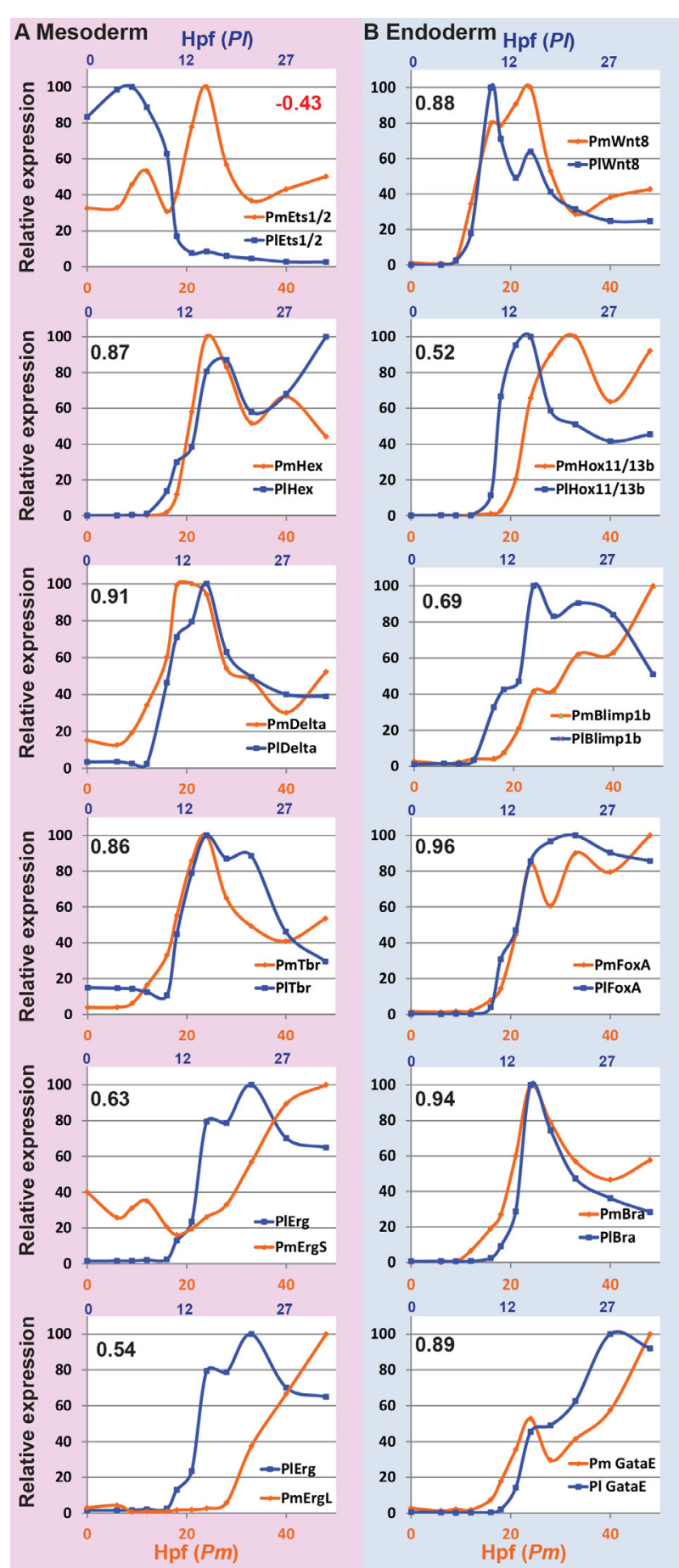
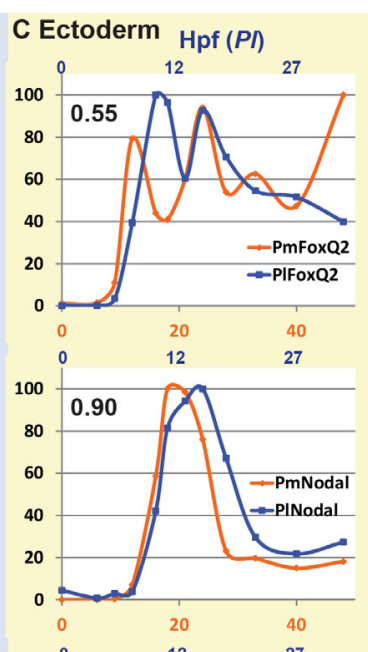

the activation of bra by low levels of nuclear $\beta$-catenin in sea stars (Mccauley et al., 2015), while in the sea urchin this gene requires additional inputs (Peter and Davidson, 2011b). The expression level of foxa is much lower than of the other endodermal genes in the sea star, while in the sea urchin it is the highest expressed endodermal gene.

The sequence of gene activation is relatively conserved in the ectoderm, where foxq2 is followed by the orderly activation of nodal, six 3 and bmp2/4 in the sea urchin and sea star (Fig. $2 \mathrm{~J}, \mathrm{~L}$, Table 1). The gene that encodes the transcription factor Foxg is activated relatively earlier in the sea star due to its early expression in the mesoderm that is absent in the sea urchin. Change is observed in the ratio between the maternal and zygotic expression level of the gene that encodes the transcription factor $h n f 6$, which is highest in late zygotic stages in the sea urchin while in the sea star it decreases at these stages. This gene is a key to the patterning of the ciliary band that shows differences between the two organisms (Yankura et al., 2013).

Thus, we observe changes in the order of gene activation (heterochronies) between the sea urchin and the sea star within the mesoderm and endoderm circuits, and evolutionary changes of the relative expression level between the maternal and zygotic stages (heterometries) in maternally expressed genes that are zygotically expressed in the mesoderm and ectoderm.
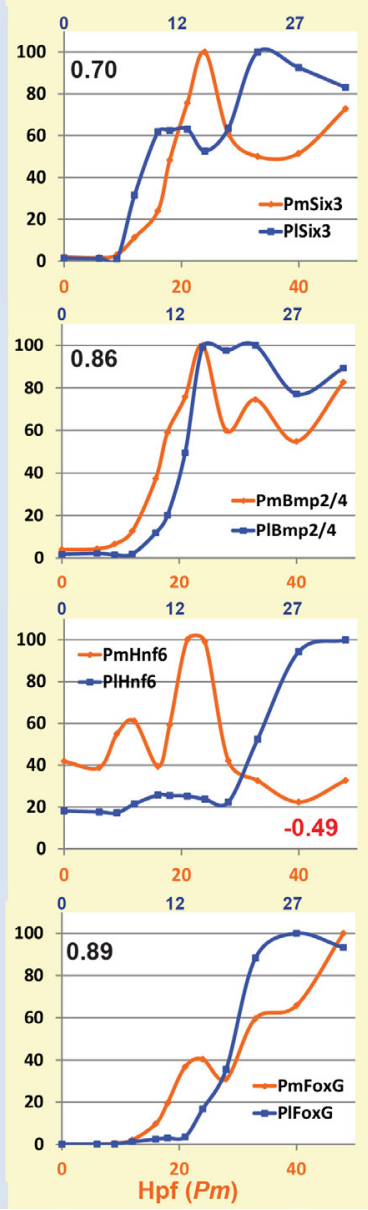
and $\mathrm{P}$. lividus developmental rates

Despite the observed changes in the order of gene activation within the circuits, most of the studied regulatory genes are initially activated in a typical developmental window in each species, which is about seven hours in the sea urchin and ten hours in the sea star (Figs. 2, 3 ). This crude scale gives a ratio of about $\times 1.4$ between the developmental rates of the sea star compared to the sea urchin. For a more accurate estimate of the relationship between the developmental rates of the two species, we used linear regression to find a linear function that relates gene initiation times in $P$. miniata to those in P. lividus, (Fig. 3A). For comparison, we made a similar estimate relating gene initiation times between two sea urchin species, P. lividus and $S$. purpuratus. These two species shared a common ancestor about 40 million years ago

Fig. 4.Temporal scaling of developmental rates and expression level normalization of $\boldsymbol{P}$ minata and $\boldsymbol{P}$. lividus regulatory genes. Corresponding developmental time points in the two species are provided in Table 3. (A) Mesoderm circuit; (B) endoderm circuit; (C) ectoderm circuit. Orange curves and time points portray P. miniata expression profiles and blue curves and time points portray P. lividus expression profiles. Numbers indicate Pearson correlation between $\mathrm{P}$. miniata and P. lividus expression profiles.
Loose temporal scaling between P. miniata 
and show high morphological similarity (Gildor and Ben-Tabou De-Leon, 2015). S. purpuratus temporal expression profiles were obtained from Materna et al., 2010. The results demonstrate the widening of the distribution of gene initiation times between the sea star and the sea urchin compared to the strict linear temporal scaling between the two sea urchin species (compare Fig. 3A and B).

The linear regression function recapitulates both the delay in the maternal to zygotic transition and the difference in zygotic gene activation between the sea urchin and the sea star: $T_{P m}=5.2+1.3 \times T_{P l}$. That is, after the $\sim 5.2$ hours delay in the maternal to zygotic transition, the molecular developmental rate in $P$. miniata is slower by about $\times 1.3$ of the $P$. lividus developmental rate. Similarly, the linear regression between the two sea urchin species shows the shorter delay of maternal to zygotic transition (2.5 hours, (Gildor and Ben-Tabou De-Leon, 2015; Gildor et al., 2016)) and a faster developmental rate compared to the sea star: $T_{S p}=2.5+T_{P l}$. The low coefficient of determination $\left(\mathrm{R}^{2}=0.27\right)$ in the sea star-sea urchin linear regression is in agreement with the broad distribution of the initiation rates of the two organisms, in particular compared to that between the two sea urchins $\left(R^{2}=0.68\right)$. The Pearson correlation between gene initiation times in the sea star and the sea urchin is, $r=0.3$, which is much lower than between the two sea urchin species, $r=0.87$. Thus, after 500 million year of divergence the exact order of gene activation is not conserved between the sea urchin and the sea star, but the overall window of gene activation shows general scaling between these two species.

Scaling the developmental rates and expression levels demonstrates heterochronies and heterometries between sea urchin and sea star

We used the linear function that relates the developmental rate in the sea star to that of the sea urchin to scale the time points in the two species, and plotted the normalized expression levels of ortholog genes one next to the other, Fig. 4. This temporal scaling and level normalization makes it easier to visually detect heterochronies and heterometries between the two species. It also enables us to calculate Pearson correlation between the expression profiles in the two species to quantify the similarities in gene temporal kinetics throughout the temporal window studied (numbers within each graph in Fig. 4).

Heterochronies in both directions are observed in all embryonic territories and regulatory circuits: the genes delta, gatae, bra, bmp2/4 and foxg are activated relatively earlier in the sea star compared to the sea urchin while the genes erg, hox11/13b, blimp1 and six 3 show the opposite trend (Fig. 4). Yet, for most genes, Pearson correlations are relatively high, in agreement with the largely similar kinetic trends in the developmental window we study. Interestingly, the highest Pearson correlations and the highest average of Pearson correlation are measured in the endoderm, the most conserved territory of the three (average Pearson correlation: Mesoderm, 0.56, Endoderm 0.81, ectoderm 0.57).

\section{Discussion}

High morphological similarity between closely related species is apparently supported by conservation of gene initiation times and order of gene activation within regulatory circuits and more loosely, within developmental transcriptomes (Gildor and Ben-Tabou DeLeon, 2015; Kalinka et al., 2010; Levin et al., 2012; Yanai et al.,
2011). At the other extreme, highly diverged body plans at large evolutionary distances are thought to emerge due to major GRN rewiring that dramatically changes both spatial and temporal expression patterns (Erwin and Davidson, 2009; Peter and Davidson, 2011a). Here we compared gene expression kinetics between two echinoderm species that show both morphological similarities and differences after 500 million years of independent evolution (Fig. 1 ). We reveal changes in the order of gene activation within the endoderm and mesoderm regulatory circuits (Fig. 2). These relative timing changes result in broad distribution of the ratios between gene expression initiations in the two species compared to the narrow distribution and fine temporal scaling between two closely related sea urchin species (Fig. 3A, B). Scaling the developmental rate of the sea urchin to that of the sea star reveals heterochronies in both directions, in all tested regulatory circuits, regardless of morphological conservation (Fig. 4). Possibly, genetic drift over 500 million years of divergence has overcome the developmental selection that enforces tight conservation of gene initiation timing and expression kinetics in closely related species. Strikingly, the highest similarity of the overall kinetic profiles, as measured by Pearson correlation, is observed at the endodermal territory, which is the most developmentally conserved lineage.

It is important to note that the interspecies divergence we see in the mRNA expression profiles of some genes could be masked at the protein level. A recent quantitative study of developmental transcriptomes and proteomes in Xenopus embryos shows that genes with highly dynamic mRNA expression profiles exhibit much more stable protein expression levels (Peshkin et al., 2015). In addition, evolutionary changes in gene translation rate, mRNA and protein degradation rates could either enhance or reduce the interspecies differences in protein expression kinetics. We believe that the interspecies protein levels may be even more conserved than the observed mRNA levels, but this conjecture requires comparative proteomic studies.

In this study we used an approach we developed before to study temporal scaling between two closely related sea urchin species (Gildor and Ben-Tabou de-Leon, 2015). In this previous study we fixed the interception point at Ohpf while here we did not fix the interception, and instead let it be defined by the best fit. As a result, the linear regression function includes the delay due to the observed shift in the maternal to zygotic transition between the species (Fig. 3 ). We believe this is a better description of the actual temporal scaling between the two species as it improves the fit parameters and agrees better with the biological processes it describes. Further expansion of this approach to entire developmental transcriptomes could help to estimate the ratio of developmental rates and distinguish between temporal scaling and heterochronies between any two organisms, whether they are closely related or further apart.

The genes we studied are expressed in equivalent spatial domains with similar GRN circuitry (Fig. 2). This similarity however, emerges following divergent maternal and early zygotic processes, which could explain the multiple heterochronies in gene initiation time we observe. The sea urchin mesoderm is specified downstream of an early "double negative gate", in which the transcription factor Pmar1 represses the global repressor HesC in the skeletogenic mesodermal cells (Oliveri et al., 2008). The suppression of HesC leads to the zygotic activation of many genes within the skeletogenic mesoderm, including delta, tbr and ets 1/2 studied here (Oliveri et al., 2008). In the sea star genome there is no evidence of a 
TABLE 2

\section{QPCR PRIMERS USED FOR THE STUDY OF P. MINIATA AND P. LIVIDUS TEMPORAL PROFILES}

\begin{tabular}{lll} 
Gene & FORWARD PRIMERS & REVERS PRIMERS \\
\hline PM_ErgS & CAGCGTGACCAAGAGACAAC & GGCACAATGACCCGTTTCTC \\
PM_Bra & AACGCCATGTACTCCATCCT & ACGCTTTGCTTCATCCAGTG \\
PM_FoxA & GCCTACCATCCAGCTACCTC & GACATCCCGTTGGTGTAGGA \\
PM_Hex & ACCCTCACATGAACCACCAT & CAAGCGGCTCCCTAGGATAT \\
PM_Hox11_13 & ATCGGCAATTCACCCATCAC & TGGAGCCGAACCCATTACTT \\
PM_GataE & CACCAATCCCCTCCTGAGTG & AAGGCTGACGATCCAAGTGA \\
PM_ErgL & TGCAAGAACTTACTCACGCG & GGTGTTGGTAGGTGCTCTCA \\
PM_Blimp1 & CGCGTTTGGACCCTTGATA & GCCTCATCCAGTTGCTCTTG \\
PM_Delta & AACAGAGCGGTTCGAATTGG & CGCATGTATTCGAGCAAGCA \\
PM Hnf6 & ATGAACGGCTCCAATCTCCA & GGATTGATGGGGTGCGTAAC \\
PM BMP2/4 & GACACACAAGGACAGAGCAC & CGGTCTGCGCATTGTAAAGA \\
PM Nodal & GCTTCCAATCACAGCTCACC & ACAGTAGCAGCGAAACTCCT \\
PM FoxG & CGCCTTTCAGTTACAACGCT & AGGTTGTGACGGATGGAGTT \\
PM Six3 & TTCTCTGGTCTCTTCCCGTG & CGTGCGATTCCTTGGTGAAT \\
PM Tbr & AACAAGGACGGCGAATGTTC & GGTACTCCATCGGACTTCCC \\
PM FoxQ2 & GAAAAGGCTCCCTCCAAAGT & GATGTCGCAGAGGAGGAGAC \\
PM_Wnt8 & CAAGAACTGCTCCGTTGGAC & CTCCATCCCATCCACGAAGA \\
PM_Ets1/2 & TTACCACAGCCACAGTCACA & ACGTCGACCATTCTGCTGTA \\
pl_Erg & CTACACCTGAGGAGCATCG & ACCCACTGCTGTACATGCTC \\
pl Hnf6 & CTCGCTTTTCACTCACGCTT & TGTGTTCTGCGATGAGTCAC \\
pl FoxG & TTAGAATGGACTCGCCACCT & TCGTGCTTCGAGTCATGTTC \\
pl Six3 & CAAACCTTCGCTACTTCGGG & CAAACCTTCGCTACTTCGGG \\
pl Tbr & CGCATACAATCACACCTGGG & ACAGACTATCTCGGAGTGCG \\
GFP & AGGGCTATGTGCAGGAGAGA & CTTGTGGCCGAGAATGTTTC \\
\hline
\end{tabular}

pmar1 gene; furthermore, HesC does not function as a repressor of mesodermal gene expression (Mccauley et al., 2010). In sea star, the mesodermal gene expression is instead initiated by high levels of nuclear $\beta$-catenin, while lower levels initiate endodermal gene expression (McCauley et al., 2015). In the sea urchin, Pmar1 is activated by $\beta$-catenin-Tcf switch, but the other mesodermal genes are not under direct control of this mechanism (Smith and Davidson, 2009). After these initially divergent activation functions, which could explain some of the observed heterochronies in gene activation, there is a distinct similarity of regulatory circuitry (Fig. 2). Indeed, after the initial heterochronies the temporal kinetics show

TABLE 3

MATCHING TIME POINTS BETWEEN P. LIVIDUS AND P. MINIATA

\begin{tabular}{cc} 
P. miniata & P. lividus \\
\hline 0 & 0 \\
6 & 2 \\
9 & 4 \\
12 & 5 \\
16 & 8 \\
18 & 10 \\
21 & 12 \\
24 & 15 \\
28 & 18 \\
33 & 22 \\
40 & 27 \\
48 & 30
\end{tabular}

higher interspecies similarity, in accordance with the activation of these more conserved circuits' linkages, especially in the highly conserved endodermal circuit (Fig. 5, (Hinman et al., 2003)). We speculate that the conservation of these regulatory circuits may be due to stabilizing selection for these later temporal profiles, which in turn are required for the activation of downstream differentiation genes. These data are also consistent with recent studies showing that gene expression states within a phylum are most similar at the mid developmental phase (Levin et al., 2016). This recent work, as well as this study, is in agreement with the hourglass model that suggests that intermediate developmental stages are most resistant to evolutionary changes within the phylum compared to earlier and later stages (Irie and Kuratani, 2011; Kalinka et al., 2010; Raff, 1996).

\section{Materials and Methods}

\section{Embryo cultures}

Adult sea urchins were supplied from a mariculture facility of the Israel Oceanographic and Limnological research in Eilat. Three independent biological repeats from three different pairs of parents were used to measure transcripts kinetics in $P$. lividus. Sea urchin eggs and sperm were obtained by injecting adult sea urchin with $0.5 \mathrm{M} \mathrm{KCl}$. Embryos were cultured at $18^{\circ} \mathrm{C}$ in artificial sea water. Sea stars were obtained in Long Beach, California, from Peter Halmay. Three independent biological repeats from three different pairs of parents were used to measure transcripts kinetics in $P$. miniata. Embryos were cultured at $15^{\circ} \mathrm{C}$ in artificial sea water.

\section{RNA extraction}

For both species, total RNA was extracted using Qiagen mini RNeasy kit from embryos at indicated time points. $1 \mu \mathrm{g}$ of total RNA from each time point of each three independent biological replicates of each species was used to generate cDNA using Applied Biosystems kit and subsequently used for QPCR.

\section{qPCR}

We designed QPCR primers based on the published $P$. lividus transcriptome (Gildor et al., 2016a) and P. miniata genome in echinobase: http:// www.echinobase.org/Echinobase/Search/PmSearch/for each gene using Primer3 web site http://primer3.ut.ee/. Primers list is provided in Table 2. The size of the amplicons was $140-160$ bp long. QPCR reactions were executed in 384-well plates using 384CFX-real time machine (BioRad). Each reaction was run in experimental triplicate and biological triplicate, hence leading to about nine measurements per gene for each time point. Every reaction contained $5 \mu \mathrm{l}$ SYBR Green mix from BioRad including $2.5 \mu \mathrm{l}$ of $1.2 \mu \mathrm{M}$ forward and reverse genes specific primers and $2.5 \mu \mathrm{l}$ of cDNA (diluted 1:50 for each assay). Thermal cycling parameters were $95^{\circ} \mathrm{C}$ for 3 min (one cycle) and then $95^{\circ} \mathrm{C}$ for $10 \mathrm{~s}, 55^{\circ} \mathrm{C}$ for $30 \mathrm{~s}$ ( 40 cycles), followed by a denaturation step to verify the amplification of a single product. To quantify the relative mRNA level we inserted a known number of GFP DNA molecules to each sample. The calculation of gene prevalence compared to GFP was performed using the formula GFP $\times 1.9^{\text {(CtGFP-CtGene) }}$, with a constant coefficient efficiency factor, 1.9, corresponding to the average value of all measured sets of primers. In our experiments we used $\sim 650,000$ GFP molecules per sample.

\section{Data analysis}

Initiation times, t0, were estimated by the use of the sigmoid function: $\log (m R N A(t))=a-b /(1+\exp (c(t-t 0))$ (Gildor and Ben-Tabou de-Leon, 2015). Half-life time, $\mathrm{t}_{1 / 2}$, of maternal genes was estimated by the use of the sigmoid function:

$m R N A(t)=a+\frac{b}{1+\exp \left(c\left(t-t_{1 / 2}\right)\right)}$ (Gildor et al., 2016). 
The sigmoid was fit using Matlab's Curve Fitting Toolbox, using the nonlinear least-squares method. Linear regression and Pearson correlations were calculated using excel Tredline and Correl functions.

\section{Acknowledgements}

We thank Dr. Greg Cary for his help in identifying P. miniata gene sequences. We thank Muki Shpigel and David Ben-Ezra for their help with sea urchin handling. This work was supported by Binational Science Foundation grant number 2015031 to SBD and VFH and the National Science Foundation IOS-0844948 to VFH.

\section{References}

ABRAHANTE, J.E., MILLER, E.A., ROUGVIE, A.E. (1998). Identification of heterochronic mutants in Caenorhabditis elegans. Temporal misexpression of a collagen:::green fluorescent protein fusion gene. Genetics 149: 1335-1351.

ADOMAKO-ANKOMAH, A., ETTENSOHN, C.A. (2013). Growth factor-mediated mesodermal cell guidance and skeletogenesis during sea urchin gastrulation. Development 140: 4214-4225.

BARSI, J.C., DAVIDSON, E.H. (2016). cis-Regulatory control of the initial neurogenic pattern of onecut gene expression in the sea urchin embryo. DevBio/409:310-318.

BARSI, J.C., LI, E., DAVIDSON, E.H. (2015). Geometric control of ciliated band regulatory states in the sea urchin embryo. Development 142: 953-961.

BEN-TABOU DE-LEON, S., SU, Y.H., LIN, K.T., LI, E., DAVIDSON, E.H. (2013). Gene regulatory control in the sea urchin aboral ectoderm: spatial initiation, signaling inputs, and cell fate lockdown. Dev Biol 374: 245-254.

BEN-TABOU DE-LEON, S., DAVIDSON, E.H. (2010). Information processing at the foxa node of the sea urchin endomesoderm specification network. Proc Natl Acad Sci USA 107: 10103-10108.

CROCE, J.C., MCCLAY, D.R. (2010). Dynamics of Delta/Notch signaling on endomesoderm segregation in the sea urchin embryo. Development 137: 83-91.

CUI, M., SIRIWON, N., LI, E., DAVIDSON, E.H., PETER, I.S. (2014). Specific functions of the Wnt signaling system in gene regulatory networks throughout the early sea urchin embryo. Proc Natl Acad Sci USA 111: E5029-5038.

DE-LEON, S.B.T., DAVIDSON, E.H. (2007). Gene regulation: Gene control network in development. Ann. Rev. Biophys. Biomolec. Struct. 36: 191-212.

DE BEER, G.R., 1930. Embryology and Evolution. Clarendon Press, Oxford.

DULOQUIN, L., LHOMOND, G., GACHE, C. (2007). Localized VEGF signaling from ectoderm to mesenchyme cells controls morphogenesis of the sea urchin embryo skeleton. Development 134: 2293-2302.

ERWIN, D.H., DAVIDSON, E.H. (2009). The evolution of hierarchical gene regulatory networks. Nat Rev Genet 10: 141-148.

GILDOR, T., MALIK, A., SHER, N., BEN-TABOU DE-LEON, S. (2016). Mature maternal mRNAs are longer than zygotic ones and have complex degradation kinetics in sea urchin. Dev Biol 414: 121-131.

GILDOR, T., MALIK, A., SHER, N., AVRAHAM, L., BEN-TABOU DE-LEON, S. (2016a). Quantitative developmental transcriptomes of the Mediterranean sea urchin Paracentrotus lividus. Marine genomics 25: 89-94.

GILDOR, T., BEN-TABOU DE-LEON, S. (2015). Comparative Study of Regulatory Circuits in Two Sea Urchin Species Reveals Tight Control of Timing and High Conservation of Expression Dynamics. PLoS Genet 11: e1005435.

GOULD, S.J., 1977. Ontogeny and Phylogeny. The Belknap Press of Harvard Uniersity Press, Cambridge, Massachusetts, USA.

GUNTER, H.M., KOPPERMANN, C., MEYER, A. (2014). Revisiting de Beer's textbook example of heterochrony and jaw elongation in fish: calmodulin expression reflects heterochronic growth, and underlies morphological innovation in the jaws of belonoid fishes. Evodevo 5: 8.

HAECKEL, E. (1875). Die Gastrula and die Eifurchung der Thiere. Jena Z. Naturwiss 9: $402-508$

HINMAN, V.F., DAVIDSON, E.H. (2007). Evolutionary plasticity of developmental gene regulatory network architecture. Proc Natl Acad Sci USA 104: 19404-19409.

HINMAN, V.F., NGUYEN, A.T., CAMERON, R.A., DAVIDSON, E.H. (2003). Developmental gene regulatory network architecture across 500 million years of echinoderm evolution. Proc Natl Acad Sci USA 100: 13356-13361.
IRIE, N., KURATANI, S. (2011). Comparative transcriptome analysis reveals vertebrate phylotypic period during organogenesis. Nat Commun 2: 248.

KALINKA, A.T., VARGA, K.M., GERRARD, D.T., PREIBISCH, S., CORCORAN, D.L., JARRELLS, J., OHLER, U., BERGMAN, C.M., TOMANCAK, P. (2010) Gene expression divergence recapitulates the developmental hourglass model. Nature 468: 811-814.

LEE, P.Y., DAVIDSON, E.H. (2004). Expression of Spgatae, the Strongylocentrotus purpuratus ortholog of vertebrate GATA4/5/6 factors. Gene Expr Patterns 5: 161-165.

LEVIN, M., ANAVY, L., COLE, A.G., WINTER, E., MOSTOV, N., KHAIR, S. SENDEROVICH, N., KOVALEV, E., SILVER, D.H., FEDER, M., FERNANDEZVALVERDE, S.L., NAKANISHI, N., SIMMONS, D., SIMAKOV, O., LARSSON, T., LIU, S.Y., JERAFI-VIDER, A., YANIV, K., RYAN, J.F., MARTINDALE, M.Q., RINK, J.C., ARENDT, D., DEGNAN, S.M., DEGNAN, B.M., HASHIMSHONY, T., YANAI, I. (2016). The mid-developmental transition and the evolution of animal body plans. Nature 531: 637-641.

LEVIN, M., HASHIMSHONY, T., WAGNER, F., YANAI, I. (2012). Developmental milestones punctuate gene expression in the Caenorhabditis embryo. Dev Cell 22: 1101-1108.

LI, E., CUI, M., PETER, I.S., DAVIDSON, E.H. (2014). Encoding regulatory state boundaries in the pregastrular oral ectoderm of the sea urchin embryo. Proc Natl Acad Sci USA 111: E906-913.

LI, E., MATERNA, S.C., DAVIDSON, E.H. (2012). Direct and indirect control of oral ectoderm regulatory gene expression by Nodal signaling in the sea urchin embryo. Dev Biol 369: 377-385.

LOGAN, C.Y., MILLER, J.R., FERKOWICZ, M.J., MCCLAY, D.R. (1999). Nuclear beta-catenin is required to specify vegetal cell fates in the sea urchin embryo. Development 126: 345-357.

MATERNA, S.C., DAVIDSON, E.H. (2012). A comprehensive analysis of Delta signaling in pre-gastrular sea urchin embryos. Dev Biol 364: 77-87.

MATERNA, S.C., NAM, J., DAVIDSON, E.H. (2010). High accuracy, high-resolution prevalence measurement for the majority of locally expressed regulatory genes in early sea urchin development. Gene Expr Patterns 10: 177-184.

MATERNA, S.C., RANSICK, A., LI, E., DAVIDSON, E.H. (2013). Diversification of oral and aboral mesodermal regulatory states in pregastrular sea urchin embryos. Dev Biol 375: 92-104.

MCCAULEY, B.S., AKYAR, E., FILLIGER, L., HINMAN, V.F. (2013). Expression of wnt and frizzled genes during early sea star development. Gene Expr Patterns 13: $437-444$

MCCAULEY, B.S., AKYAR, E., SAAD, H.R., HINMAN, V.F. (2015). Dose-dependent nuclear beta-catenin response segregates endomesoderm along the sea star primary axis. Development 142: 207-217.

MCCAULEY, B.S., WEIDEMAN, E.P., HINMAN, V.F. (2010). Aconserved gene regulatory network subcircuit drives different developmental fates in the vegetal pole of highly divergent echinoderm embryos. Dev Biol 340: 200-208.

MCCAULEY, B.S., WRIGHT, E.P., EXNER, C., KITAZAWA, C., HINMAN, V.F. (2012) Development of an embryonic skeletogenic mesenchyme lineage in a sea cucumber reveals the trajectory of change for the evolution of novel structures in echinoderms. Evodevo 3: 17.

MORINO, Y., KOGA, H., TACHIBANA, K., SHOGUCHI, E., KIYOMOTO, M., WADA $\mathrm{H}$. (2012). Heterochronic activation of VEGF signaling and the evolution of the skeleton in echinoderm pluteus larvae. Evol Dev 14: 428-436.

OLIVERI, P., TU, Q., DAVIDSON, E.H. (2008). Global regulatory logic for specification of an embryonic cell lineage. Proc Natl Acad Sci USA 105: 5955-5962.

OTIM, O., HINMAN, V.F., DAVIDSON, E.H. (2005). Expression of AmHNF6, a sea star orthologue of a transcription factor with multiple distinct roles in sea urchin development. Gene Expr Patterns 5: 381-386.

PASQUINELLI, A.E., REINHART, B.J., SLACK, F., MARTINDALE, M.Q., KURODA M.I., MALLER, B., HAYWARD, D.C., BALL, E.E., DEGNAN, B., MULLER, P., SPRING, J., SRINIVASAN, A., FISHMAN, M., FINNERTY, J., CORBO, J., LEVINE, M., LEAHY, P., DAVIDSON, E., RUVKUN, G. (2000). Conservation of the sequence and temporal expression of let-7 heterochronic regulatory RNA. Nature 408:86-89.

PESHKIN, L., WUHR, M., PEARL, E., HAAS, W., FREEMAN, R.M., JR., GERHART, J.C., KLEIN, A.M., HORB, M., GYGI, S.P., KIRSCHNER, M.W. (2015). On the Relationship of Protein and mRNA Dynamics in Vertebrate Embryonic Development. Dev Cell 35: 383-394.

PETER, I.S., DAVIDSON, E.H. (2011a). Evolution of gene regulatory networks con- 
trolling body plan development. Cell 144: 970-985.

PETER, I.S., DAVIDSON, E.H. (2011b). A gene regulatory network controlling the embryonic specification of endoderm. Nature 474: 635-639.

RAFF, R.A., 1996. The Shape of Life: Genes. Development and the Evolution of Animal Form. The University of Chicago Press, Chicago.

RANSICK, A., DAVIDSON, E.H. (2006). cis-regulatory processing of Notch signaling input to the sea urchin glial cells missing gene during mesoderm specification. Dev Biol 297: 587-602.

ROTTINGER, E., CROCE, J., LHOMOND, G., BESNARDEAU, L., GACHE, C., LEPAGE, T. (2006). Nemo-like kinase (NLK) acts downstream of Notch/Delta signalling to downregulate TCF during mesoderm induction in the sea urchin embryo. Development 133: 4341-4353.

SAKAMOTO, K., ONIMARU, K., MUNAKATA, K., SUDA, N., TAMURA, M., OCHI, H., TANAKA, M. (2009). Heterochronic shift in Hox-mediated activation of sonic hedgehog leads to morphological changes during fin development. PLoS One 4: e5121.

SAUDEMONT, A., HAILLOT, E., MEKPOH, F., BESSODES, N., QUIRIN, M., LAPRAZ, F., DUBOC, V., ROTTINGER, E., RANGE, R., OISEL, A., BESNARDEAU, L., WINCKER, P., LEPAGE, T. (2010). Ancestral regulatory circuits governing ectoderm patterning downstream of Nodal and BMP2/4 revealed by gene regulatory network analysis in an echinoderm. PLoS Genet 6: e1001259.

SMITH, A.B., ZAMORA, S., ALVARO, J.J. (2013). The oldest echinoderm faunas from Gondwana show that echinoderm body plan diversification was rapid. Nat Commun 4: 1385.

SMITH, J., DAVIDSON, E.H. (2008). Gene regulatory network subcircuit controlling a dynamic spatial pattern of signaling in the sea urchin embryo. Proc Natl Acad Sci USA 105: 20089-20094.

SMITH, J., DAVIDSON, E.H. (2009). Regulative recovery in the sea urchin embryo and the stabilizing role of fail-safe gene network wiring. Proc Natl Acad Sci USA.

SMITH, J., THEODORIS, C., DAVIDSON, E.H. (2007). A gene regulatory network subcircuit drives a dynamic pattern of gene expression. Science 318: 794-797.

SMITH, M.S., TURNER, F.R., RAFF, R.A. (2008). Nodal expression and heterochrony in the evolution of dorsal-ventral and left-right axes formation in the direct-developing sea urchin Heliocidaris erythrogramma. J Exp Zool B Mol Dev Evol310: 609-622.

TU, Q., BROWN, C.T., DAVIDSON, E.H., OLIVERI, P. (2006). Sea urchin Forkhead gene family: phylogeny and embryonic expression. Dev Biol 300: 49-62.

VELHAGEN, W.A., JR. (1997). Analyzing developmental sequences using sequence units. Syst Biol 46: 204-210.

WEI, Z., YAGUCHI, J., YAGUCHI, S., ANGERER, R.C., ANGERER, L.M. (2009). The sea urchin animal pole domain is a Six3-dependent neurogenic patterning center. Development 136: 1179-1189.

YAGUCHI, S., YAGUCHI, J., ANGERER, R.C., ANGERER, L.M. (2008). A WntFoxQ2-nodal pathway links primary and secondary axis specification in sea urchin embryos. Dev Cell 14: 97-107.

YANAI, I., PESHKIN, L., JORGENSEN, P., KIRSCHNER, M.W. (2011). Mapping gene expression in two Xenopus species: evolutionary constraints and developmental flexibility. Dev Cell 20: 483-496.

YANKURA, K.A., KOECHLEIN, C.S., CRYAN, A.F., CHEATLE, A., HINMAN, V.F. (2013). Gene regulatory network for neurogenesis in a sea star embryo connects broad neural specification and localized patterning. Proc Natl Acad Sci USA 110: 8591-8596.

YANKURA, K.A., MARTIK, M.L., JENNINGS, C.K., HINMAN, V.F. (2010). Uncoupling of complex regulatory patterning during evolution of larval development in echinoderms. BMC Biol 8: 143. 


\section{Further Related Reading, published previously in the Int. J. Dev. Biol.}

The involvement of three signal transduction pathways in botryllid ascidian astogeny, as revealed by expression patterns of representative genes

Amalia Rosner, Gilad Alfassi, Elizabeth Moiseeva, Guy Paz, Claudette Rabinowitz, Ziva Lapidot, Jacob Douek, Abraham Haim and Baruch Rinkevich

Int. J. Dev. Biol. (2014) 58: 677-692

http://dx.doi.org/10.1387/ijdb.140114ar

What Hydra can teach us about chemical ecology - how a simple, soft organism survives in a hostile aqueous environment Tamar Rachamim and Daniel Sher Int. J. Dev. Biol. (2012) 56: 605-611 http://dx.doi.org/10.1387/ijdb.113474tr

Gene regulatory networks governing haematopoietic stem cell development and identity John E. Pimanda and Berthold Göttgens Int. J. Dev. Biol. (2010) 54: 1201-1211

\section{Genomic control of patterning}

Isabelle S. Peter and Eric H. Davidson

Int. J. Dev. Biol. (2009) 53: 707-716

\section{Developmental gene network analysis.}

Roger Revilla-i-Domingo and Eric H Davidson

Int. J. Dev. Biol. (2003) 47: 695-703
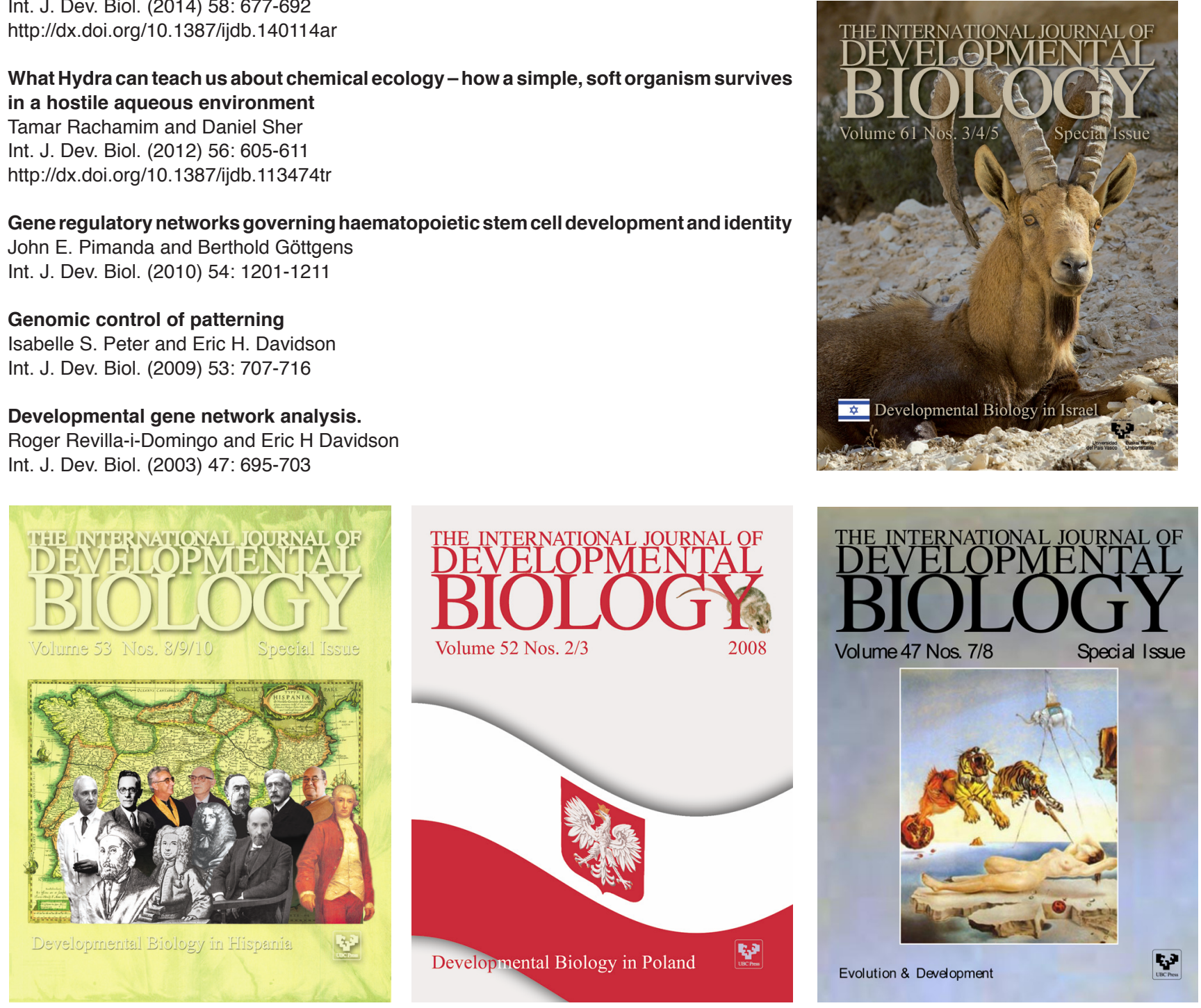